\title{
LOS PRIMEROS DOCE AÑOS DE AUTONOMÍA EN LA COMUNIDAD DE LAS ISLAS BALEARES
}

\author{
JOAN OLIVER ARAUJO \\ Catedrático de Derecho Constitucional \\ ANDREU RIBAS MAURA \\ Profesor Titular de Derecho Constitucional
}




\section{SUMARIO}

1. La creación de la Comunidad Autónoma de las islas Baleares.2. La Primera legislatura (1983-1987).-3. La Segunda Legislatura (1987-1991).-4. La Tercera LegisLatura (1991-1994).-5. Valoración DE LOS PRIMEROS DOCE ANNOS DE LA AUTONOMIA BALEAR. 
Revista de Derecho Político, núm. 38, 1994, pp. 267-278

\title{
LOS PRIMEROS DOCE AÑOS DE AUTONOMÍA EN LA COMUNIDAD DE LAS ISLAS BALEARES
}

POR

\author{
JOAN OLIVER ARAUJO \\ Catedrático de Derecho Constitucional
}

ANDREU RIBAS MAURA

Profesor Titular de Derecho Constitucional

1. LA CREACIÓN DE LA COMUNIDAD AUTÓNOMA DE LAS ISLAS BALEARES

Las Islas Baleares tuvieron que recorrer un largo, difícil y, en muchos momentos, crispado camino antes de poder contar con un Estatuto de Autonomía al amparo de la Constitución de 1978. A pesar de la decidida voluntad de autogobierno de estas tierras, manifestada tanto durante la Segunda República (aprobación de un Anteproyecto de Estatuto en 1931) como tras la etapa franquista (manifestación de más de cincuenta mil personas el 29 de octubre de 1977 pidiendo la autonomía), diversas circunstancias políticas dificultaron la formulación jurídica de tal deseo.

Una vez aprobada la Constitución, los esfuerzos de los partidos políticos se orientaron a la consecución de un Estatuto de Autonomía siguiendo las vías previstas en el Texto Constitucional. El acuerdo y el clima favorable existente hasta entonces parecían augurar una rápida consecución de tal propósito. Sin embargo, el Estatuto balear no fue aprobado definitivamente hasta finales del mes de febrero de 1983, dándose la peculiar circunstancia "de ver llegar la autonomía de un pueblo que históricamente ha sido Reino independiente, con organización políti- 
ca propia, compartiendo el furgón de cola del tren autonómico con las Comunidades de Extremadura, Madrid y Castilla-León» (M. MASOT MIQUEL).

¿Cuáles fueron los motivos que ocasionaron la dilatación del proceso autonómico balear? Sin duda influyeron varias causas, tales como las discusiones sobre la bandera, el problema de la lengua y la vía de acceso, así como la disolución de las Cortes Generales mientras se tramitaba el Estatuto. Pero -como dijo repetidamente el diputado GREGORI MIR MAYOL en las discusiones parlamentarias - fue la cuestión electoral, y en concreto el reparto de los parlamentarios regionales entre las Islas, "la auténtica cuestión de fondo, el auténtico caballo de batalla desde el año 1979" y el que "ha mantenido paralizado el proceso estatutario de las Islas durante casi cuatro años".

El iter de la Ley Orgánica 21/1983, de 25 de febrero, de Estatuto de Autonomía para las Islas Baleares ("B.O.E." de 1 de marzo de 1983), comenzó en junio de 1980. En esta fecha las fuerzas políticas de Baleares acordaron, sin prejuzgar la vía a través de la cual se accedería a la autonomía, constituir una Comisión (a nivel popular conocida como la "Comissió dels onze") con el objeto de redactar un Anteproyecto de Estatuto. Diez meses más tarde, en abril del año siguiente, la referida Comisión hizo entrega del texto elaborado al Presidente del Consell General Interinsular, quien, una vez adoptada la vía lenta del artículo 143 , convocó la Asamblea de parlamentarios y consejeros que debía elaborar y aprobar el Proyecto de Estatuto (art. 146). Dicha Asamblea, a pesar de la duras críticas que a su convocatoria dirigió Alianza Popular (por entender que no se habian cumplido todos los requisitos), se constituyó el 4 de diciembre de 1981 y tres días después aprobó el Proyecto definitivo. Este texto se entregó al Presidente de las Cortes Generales a finales de 1981, y un año más tarde, el 26 de enero de 1983, fue aprobado, con importantes enmiendas, por el Pleno del Congreso de los Diputados. Posteriormente, el texto fue remitido a la Cámara Alta, en donde recibió el respaldo de su Pleno en la sesión de 17 de febrero de 1983. Tras ser ratificado por el Congreso, el texto aprobado por el Senado adquirió valor definitivo.

\section{LA PRIMERA LEGISLATURA (1983-1987)}

El artículo 20 del Estatuto de Autonomía dejaba a una futura ley del Parlamento balear la regulación de los aspectos electorales básicos. Sin embargo, para llenar este vacio normativo, la disposición transitoria segunda b) estableció unas normas provisionales a aplicar en la primera 
elección de la Cámara Legislativa regional. Esta regulación transitoria se concretaba en los siguientes puntos: 1.9) el número total de diputados a elegir se cifraba en cincuenta y cuatro; 2.9) se dividía la Comunidad Autónoma en cuatro distritos electorales, correspondientes a las cuatro Islas habitadas del Archipiélago: Mallorca elegía 30 diputados, Menorca 12, Ibiza 11 y Formentera 1.

De acuerdo con el contenido de esta disposición transitoria, el 8 de mayo de 1983 se celebraron las primeras elecciones al Parlamento balear, produciéndose los siguientes resultados: la Coalición Popular (APPDP-UL) y el Partido Socialista Obrero Español obtuvieron 21 escaños cada uno; Unió Mallorquina, 6; el Partit Socialista de Mallorca-Partit Socialista de Menorca, 4, y la Candidatura Independent de Menorca y el Partido Demócrata-Liberal (Ibiza-Formentera), 1 cada uno. En resumen, pues, la derecha logró 21 escaños, el centro-derecha 8 y la izquierda 25.

El día 31 de mayo tuvo lugar la sesión inaugural del Parlamento balear, y el día 7 del mes siguiente Gabriel Cañellas Fons, líder de Alianza Popular, fue elegido, con el apoyo de la derecha y el centro, primer Presidente de la Comunidad Autónoma de las Islas Baleares. Un Gobierno formado exclusivamente por conservadores, aunque con el inicial apoyo parlamentario de los regionalistas, dirigiría, con notables dificultades, los primeros cuatro años (1983-1987) de la senda autonómica balear. En efecto, el Ejecutivo conservador no pudo gobernar en muchos momentos a causa de la ambigua actitud de Unió Mallorquina, partido dirigido por Jeroni Albertí Picornell. Así, aunque en principio los regionalistas firmaron un pacto de legislatura con Coalición Popular (lo que permitió a Albertí alcanzar la presidencia del Consell Insular de Mallorca), Unió Mallorquina, como reconoció el propio Albertí (diario Baleares, 24 de junio de 1986), fue dando "bandazos", careciendo de una "personalidad definidan.

En esta Primera Legislatura, la Comunidad Autónoma fue dotándose de los instrumentos jurídico-políticos precisos para establecer su organización interna y asegurar su buen funcionamiento; asimismo, fue asumiendo los primeros bloques de transferencias de competencias

Por lo que se refiere a los aspectos organizativos, destacan las dificultades para la elaboración y aprobación de la normativa electoral. En efecto, tras grandes polémicas en torno a la conveniencia de adoptar un sistema paritario (número de diputados de Mallorca = número de diputados de las tres Islas menores) o proporcional, se llegó a aprobar la Ley Electoral de la Comunidad Autónoma de las Islas Baleares (Ley 8/1986, de 26 de noviembre). Curiosamente, el Proyecto - de carácter paritariopresentado por el Gobierno conservador fue rechazado por el Parlamen- 
to, al aceptar una enmienda a la totalidad con texto alternativo presentada por el Grupo Parlamentario Socialista, que recogía el sistema proporcional aunque fuertemente corregido (el número total de diputados que integran el Parlamento pasa a ser de 59: Mallorca elige 33, Menorca 13, Ibiza 12 y Formentera 1). Esta derrota gubernamental pudo darse porque los parlamentarios de Unió Mallorquina, excepcionalmente, votaron con la oposición de izquierdas. Se produjo de esta forma el hecho sin precedentes de ver cómo el Parlamento balear rechazaba una ley fundamental presentada por el Gobierno y aprobaba por mayoría absoluta la propuesta presentada por el principal grupo de la oposición. La polémica, sin embargo, no se cerró con la aprobación de dicha Ley. Por el contrario, cincuenta y seis diputados de la Coalición Popular -instados, sobre todo, por destacados conservadores ibicencos- interpusieron recurso de inconstitucionalidad contra la misma. No obstante, cuando el Tribunal Constitucional, por Sentencia 45/1992, de 2 de abril, desestimó por unanimidad dicho recurso, no se suscitó ya polémica alguna.

Junto a las controversias relacionadas con los temas electorales, en esta Primera Legislatura podemos registrar otras cuestiones de notable relevancia política. En primer lugar, la cuestión de confianza presentada - y ganada - por el Presidente del Gobierno para obligar al grupo regionalista a salir de su ambigüedad y a manifestarle expresamente su apoyo (las diferencias entre Coalición Popular y Unió Mallorquina en el tema de la protección de los espacios naturales ya empezaban a ser importantes). En segundo lugar, deben destacarse las acusaciones de actuación irregular que la oposición dirigió contra el Ejecutivo. En relación a este último punto, debemos indicar que el Parlamento balear creó dos comisiones de investigación. La primera tenía por objeto investigar unas presuntas irregularidades e incompatibilidades del Presidente de la Comunidad Autónoma con motivo de contratas para obras en edificios de la Comunidad; y la segunda tenía por cometido investigar unas presuntas irregularidades en materia de contratación de personal al servicio de la Comunidad Autónoma.

\section{LA SEGUNDA LEGISLATURA (1987-1991)}

Las elecciones autonómicas que se celebraron el día 10 de junio de 1987 iniciaron, de hecho, la Segunda Legislatura del Parlamento balear. En estos comicios, los resultados en escaños fueron los siguientes (debe tenerse en cuenta que la Cámara Legislativa había ampliado su composición, pasando de 54 a 59 diputados): Alianza Popular obtuvo 25, el Partido Socialista Obrero Español 21, el Centro Democrático y Social 5, Unió 
Mallorquina 4 y el Partit Socialista de Mallorca-Partit Socialista de Menorca 4. Una breve valoración de estos resultados nos permite hacer las siguientes afirmaciones: 1. ${ }^{\text {a) }}$ Alianza Popular había aumentado moderadamente los resultados obtenidos en las anteriores elecciones autonómicas (de 21 a 25 escaños, aunque este aumento debe ponderarse teniendo en cuenta la ampliación de la Cámara), lo cual daba a entender que los cuatro años de gobierno, lejos de debilitar al partido conservador, le consolidaban como el primer partido de la Comunidad. 2.9) EI PSOE experimentaba un ligero retroceso en sus posiciones (pues mantenía el mismo número de escaños -21- en un Parlamento más numeroso). 3.9) EI CDS hacía una fuerte aparición en el panorama parlamentario balear, y pudo haberse convertido en la clave para cualquier gobierno estable. 4.a) Unió Mallorquina sufrió un fuerte castigo electoral, debido posiblemente a su escasa coherencia política y a su ambigua estrategia en la Primera Legislatura. 5.9) Los nacionalistas de izquierdas mantuvieron sus posiciones, obteniendo dos diputados por Mallorca y dos por Menorca.

Estos resultados en las elecciones del 10 de junio de 1987 dejaban abiertas tanto las posibilidades de un gobierno conservador como las de un gobierno progresista. En efecto, los partidos de derechas (AP) y de centro-derecha (UM) tenían 29 escaños y los partidos de izquierdas (PSOE y PSM-PSME) tenían 25. Ninguna de las dos opciones tenía los treinta escaños de la mayoría absoluta $y$, por tanto, los cinco diputados del Centro Democrático y Social iban a ser, en principio, los árbitros de la situación. Tanto el PSOE como AP ofrecieron al CDS iniciar conversaciones con vistas a formar un gobierno de coalición; sin embargo, siguiendo las consignas de Madrid, los hombres de Suárez en Baleares optaron por quedarse al margen del gobierno y abstenerse en la votación de investidura, dejando que gobernase el partido que había obtenido la mayoría relativa. De esta manera, Alianza Popular y Unió Mallorquina (que, al dejar de tener los escaños suficientes para ser decisiva su colaboración en el gobierno, había minimizado sus diferencias con el partido conservador) formaron un gobierno minoritario, que sin embargo estaba a un solo escaño de obtener la mayoría absoluta. Gabriel Cañellas Fons, Presidente de AP, repetiría como Presidente del Ejecutivo, mientras que Jeroni Albertí Picornell, Presidente de UM, accedería a la Presidencia del Legislativo Autonómico. A cambio de su abstención en la votación de investidura, el Centro Democrático y Social, que como hemos dicho tenía la llave de todas las decisiones importantes, obtuvo el senador autonómico y la Vicepresidencia Primera del Parlamento.

Éste era el panorama inicial de la legislatura hasta que, al poco de iniciarse, el diputado del CDS por Menorca Carlos Ricci abandonó su partido político, alegando que había incumplido el programa electoral, y se integró en el grupo mixto (de hecho, se creó para él y él era su único in- 
tegrante). Ricci, acusado de oportunista y de mercader de la política por buena parte de los partidos y de los medios de comunicación, dio su apoyo al gobierno conservador, convirtiéndose en el voto número 30 que le faltaba para tener la mayoría absoluta. De ser un oscuro diputado de un partido minoritario, Ricci se convirtió en la "auténtica estrella" de esta legislatura (el "chico treinta", como le apodó la prensa de estos años), pues su voto dio la mayoría absoluta al gobierno, disipó cualquier posibilidad de gobierno de izquierdas y convertía a su antiguo partido -el CDS-en un mero comparsa, cuando hasta entonces tenía un protagonismo sobresaliente.

El Gobierno conservador -con el apoyo parlamentario de los diputados de Alianza Popular, Unió Mallorquina y del tránsfuga Carlos Ricci- dirigió la política de nuestra Comunidad entre los años 1987-1991 sin especiales dificultades por lo que se refiere a su estabilidad.

De forma casi telegráfica, podemos resumir en los siguientes términos algunas de las cuestiones políticas más relevantes de la Segunda Legislatura del Parlamento balear. En primer lugar, debe destacarse que esta legislatura vino marcada por el hecho cronológico de que en ella se cumplían los cinco años que, de acuerdo con el artículo 148.2 de la Constitución, permitían reformar el Estatuto de la Comunidad Autónoma de las Islas Baleares para ampliar sus competencias, hasta equipararse a las Comunidades que habían accedido a la autonomía por la vía rápida. Así, el 10 de noviembre de 1988 el Pleno del Parlamento se pronunció favorablemente a la mencionada ampliación. En segundo lugar, hay que subrayar que, tras repetidos intentos, se aprobó al fin la Ley de los Consejos Insulares (Ley 5/1989, de 13 de abril). Esta norma resultaba imprescindible para la completa configuración del sistema autonómico balear, pues los Consejos Insulares (Consells Insulars), respetando las competencias propias del Gobierno y el Parlamento de la Comunidad, tienen asignados estatutariamente el gobierno, la administración y la representación de las islas de Mallorca, Menorca e lbiza-Formentera. En tercer lugar, debe dejarse constancia de la progresiva crisis de Unió Mallorquina, lo que llevaría a que su Presidente y fundador, Jeroni Albertí, la abandonara y a que estuviera al borde mismo de la desaparición (sólo evitada, in extremis y de momento, gracias a un pacto preelectoral con el Partido Popular en 1991). Finalmente, en cuarto lugar, merece remarcarse la dura polémica que acompañó la elaboración y aprobación de la Ley de Espacios Naturales (la cual iba a determinar qué parte de nuestras Islas iba a quedar protegida y qué parte podía ser objeto de urbanización). En este punto, la posición de los partidos era, en síntesis, la siguiente: el PSOE, el CDS (a excepción del diputado Tuells) y el PSM-PSME defendían la opción proteccionista; Alianza Popular y Unió Mallorquina (a excepción del diputado Miquel Pascual) estaban a favor de limi- 
tar los espacios protegidos, posibilitando una mayor edificación. Otra vez estábamos ante un 29 a 29, y otra vez el voto del diputado Carlos Ricci volvía a ser decisivo. Éste, aunque en numerosos aspectos de esta ley votó con el Gobierno, al tratarse el punto concreto e importantísimo de "Ses Salines", de Ibiza, se abstuvo en dos ocasiones, provocando dos empates a 29 votos, y finalmente, en la tercera votación, dio su apoyo a las tesis proteccionistas defendidas por la oposición de izquierdas. Inmediatamente, el Gobierno conservador hizo público un comunicado en el que afirmaba que, si obtenía la mayoría suficiente en las próximas elecciones autonómicas, modificaría la Ley que acababa de aprobarse, de acuerdo con los criterios ("construccionistas") que había defendido en el Parlamento.

\section{LA TERCERA LEGISLATURA (1991-1994)}

La tercera y actual legislatura ha supuesto la consolidación del Partido Popular en Baleares. Esto ha permitido a su Presidente, Gabriel Cañellas Fons, iniciar un tercer mandato al frente del Gobierno autonómico, al contar con el apoyo de la mayoría absoluta del Parlamento balear. En efecto, en las elecciones autonómicas, celebradas el 26 de mayo de 1991, se dieron los siguientes resultados en cuanto al reparto de diputados: Partido Popular-Unió Mallorquina 31, Partido Socialista Obrero Español 21, Partit Socialista de Mallorca-Entesa de l'Esquerra de Menorca 5, Unió Independent de Mallorca 1 y Independents d'Eivissa 1.

La clave que nos permite interpretar y valorar correctamente estos resultados es, sin duda, la coalición preelectoral entre el Partido Popular y Unió Mallorquina. Este pacto, además de las ventajas que se deducen de la pura matemática electoral, supuso una ampliación de la base sociológica de los partidos de centro-derecha, que se reflejó en un aumento del número de votos con respecto a los comicios anteriores. Sin embargo, a pesar de esta importante victoria, la coalición PP-UM ya ha tenido sus primeros altibajos en cuanto al funcionamiento efectivo del pacto de gobierno. La manifestación más importante de las tensiones se dio cuando el Presidente del Gobierno balear decretó el cese de la Consejera de Cultura y Presidenta de Unió Mallorquina. Tras un breve período de incertidumbre, las aguas volvieron, aparentemente, a su cauce al ser nombrado para tal cargo otro miembro del mismo partido coaligado.

El segundo factor a considerar se refiere a los resultados electorales de los partidos de la oposición, en especial el CDS y el PSOE. El primero, como fue la pauta general de las elecciones del 91, desapareció del 
mapa autonómico balear, dejando libre un espacio electoral que, presumiblemente, entró en la órbita de la coalición PP-UM. El segundo, aunque manteniendo el mismo número de escaños que en la legislatura anterior, sufrió un significativo descenso de votos, que se manifestó especialmente importante en el municipio de Palma.

Sin embargo, la coalición PP-UM tuvo pronto sus primeros altibajos en cuanto al funcionamiento efectivo del pacto de gobierno. La primera manifestación destacable de las tensiones se dio cuando el Presidente del Gobierno balear decretó el cese de la Consejera de Cultura y Presidenta de Unió Mallorquina (junio de 1993). Tras un breve período de incertidumbre, las aguas volvieron, aparentemente, a su cauce al ser nombrado para tal cargo otro miembro del mismo partido coaligado. No obstante, la calma duró poco tiempo, pues en abril de 1994 Unió Mallorquina rompió - por motivos, aparentemente, electorales- la coalición que mantenía con el Partido Popular. Este hecho, que provocó el abandono de algunos militantes de Unió Mallorquina, terminó con la mayoría parlamentaria que venía respaldando al Gobierno, lo cual forzó al partido conservador a buscar nuevos apoyos parlamentarios y a la reestructuración de algunas instituciones, especialmente del Consell Insular de Mallorca. De esta forma, todo volvió a quedar pendiente del voto de un diputado-tránsfuga, al producirse un nuevo empate a veintinueve escaños entre el Partido Popular y la suma de los partidos de la oposición. En esta ocasión fue el voto del tránsfuga Jaume Peralta (que había obtenido el acta de diputado en la lista del PSOE por Menorca) el que rompió el empate, uniendo su voto -en todos los casos decisivos- al del grupo conservador.

Por lo que se refiere a las cuestiones más polémicas de esta legislatura, debemos indicar que la atención política se ha centrado en buena medida en los temas que ya fueron objeto de interés en la segunda legislatura. Ahora bien, dos son las cuestiones que destacan sobre las demás:

1.") La primera cuestión gira en torno a la delimitación de los espacios naturales protegidos. En este tema, el partido mayoritario cumplió su promesa electoral de modificar la Ley de Espacios Naturales, en el sentido de reducir la protección urbanística establecida por la Ley en determinadas áreas del territorio balear. La modificación fue extremadamente polémica, tanto en el Parlamento como en la calle, y suscitó reticencias, no sólo de los grupos de la oposición, sino también del entonces socio de coalición del Partido Popular.

2.") El segundo tema incide en la política de descentralización administrativa en los Consejos Insulares, recién iniciada en las postrimerías de la Segunda Legislatura. En este ámbito, la actividad ejercida por 
los Consells en el área de urbanismo, que fue la primera materia transferida a estos entes, ya ha dado lugar a conflictos judiciales, aún abiertos, y a polémicas parlamentarias. Más recientemente (diciembre de 1993). con menor polémica, se han transferido las competencias en materia de régimen local, información turística, servicios sociales y asistencia social e inspección técnica de vehículos. La lista de materias pendientes de transferencia es, no obstante, más extensa; además, el papel de los Consejos y su relación con el Gobierno balear es un tema aún no resuelto completamente. El debate sobre cómo debe realizarse la descentralización administrativa dentro de la propia Comunidad es, pues, uno de los más importantes retos a los que ésta se enfrenta. Se trata, en definitiva, del propio diseño institucional de la Comunidad Autónoma de las Islas Baleares.

Finalmente, no puede cerrarse la reseña de esta legislatura sin hacer una breve mención a un proceso que repercutirá notablemente en la actividad autonómica y que se encuentra dando sus primeros pasos: la ampliación del techo competencial derivada de la Ley Orgánica de Transferencia de nuevas competencias por parte del Estado a las Comunidades Autónomas, en desarrollo del llamado "Pacto autonómico". Todo ello, unido a la reforma del Estatuto de Autonomía de las Islas Baleares, al objeto de recoger las nuevas competencias. Este hecho abre nuevas perspectivas en el futuro de nuestra Comunidad Autónoma.

\section{VALORACIÓN DE LOS PRIMEROS DOCE AÑOS DE LA AUTONOMÍA BALEAR}

De forma necesariamente breve y esquemática, podemos hacer la siguiente valoración de los primeros doce años de autonomía en la Comunidad de las Islas Baleares:

1.) Durante estos doce años ha estado al frente de la Comunidad un gobierno marcadamente conservador, presidido por Gabriel Canellas. Este gobierno ha recibido el apoyo parlamentario del o de los partidos de derechas (AP-PDP-UL en 1982, AP en 1987 y PP en 1991) y, con algunas reticencias, de Unió Mallorquina (al menos hasta la primavera de 1994). Hay que añadir, en esta línea, que la política clientelista y la estrategia de coalición propiciadas por el Presidente Cañellas le han proporcionado un importante rédito electoral.

2.9) Por otra parte, es fácilmente constatable que el entusiasmo inicial por la autonomía en amplios sectores de la sociedad balear (re- 
cordemos, a modo de ejemplo paradigmático, la manifestación de más de cincuenta mil personas que tuvo lugar en octubre de 1977) fue dejando paso a un rápido desencanto y a una progresiva desilusión. La comprobación de que la autonomía no es la panacea de todos los males, y ciertas prácticas políticas poco edificantes de algunos de los principales actores políticos, fueron las principales causas de este desencanto y de esta desilusión.

3.9) También ha sido una constante en esta primera etapa de la autonomía balear una importante confusión entre los ciudadanos sobre los cometidos que había asumido la Comunidad y sobre la precisa distribución de competencias entre la Comunidad Autónoma, los Consejos Insulares y los Ayuntamientos.

4.9) Es de destacar que, como era previsible, ha surgido una clase política autonómica -principalmente de derechas, aunque también de izquierdas- que ha adquirido un papel protagonista y un conjunto de privilegios de nuevo cuño.

5.9) Las relaciones entre las diferentes Islas del Archipiélago Balear - tradicionalmente muy complejas por sus notables diferencias históricas, ideológicas y estructurales, y por el recelo que inspira Mallorca a las Islas menores- han mejorado muy sensiblemente. En efecto, en estos doce primeros años de autogobierno, la Comunidad Autónoma de las Islas Baleares se ha consolidado como una Comunidad plural y armónica, en la cual los cuatro pueblos insulares se encuentran, desde su propia historia, para construir un futuro en el marco de un autogobierno común. 